\title{
Epidemiological characteristics of the 2005 and 2007 dengue epidemics in Singapore - similarities and distinctions
}

\author{
Teck Siang Ler, ${ }^{a}$ Li Wei Ang, ${ }^{b}$ Grace Siew Lian Yap, ${ }^{c}$ Lee Ching Ng, ${ }^{c}$ Ji Choong Tai, ${ }^{d}$ Lyn James ${ }^{b}$ and Kee Tai Gohe \\ Correspondence to Teck Siang Ler (e-mail: ts.ler@me.com)
}

Introduction: We investigated the epidemiological features of the 2007 dengue outbreak to determine the factors that could have triggered it two years after the previous large outbreak in 2005.

Methods: All laboratory-confirmed cases of dengue reported during the year, as well as entomological and virological data, were analysed.

Results: A total of 8826 cases including 24 deaths were reported in 2007, giving an incidence of 192.3 cases per 100000 residents and a case-fatality rate of $0.27 \%$. The median age of the cases was 37 years (interquartile range 25 to 50), with an age range from two days to 101 years, which was higher than the median age of 31 years (interquartile range 20 to 42), with a range from four days to 98 years, in 2005. The overall Aedes premises index in 2007 was $0.68 \%$, lower than the $1.15 \%$ observed in 2005 . The predominant dengue serotype in 2007 was dengue virus DENV-2 which re-emerged with a clade replacement in early 2007 , and overtook the predominant serotype (DENV-1) of 2005. Seroprevalence studies conducted in the three largest outbreak clusters revealed that $73.2 \%$ of residents with recent infection were asymptomatic.

Discussion: With the exception of an increase in the median age of the cases, and a change in the predominant dengue serotype, the epidemiological features of the 2007 epidemic were largely similar to those of 2005. Singapore remains vulnerable to major outbreaks of dengue, despite sustained vector control measures to maintain a consistently low Aedes premises index.

$\mathrm{T}$ he four serotypes of dengue virus (DENV) (family Flaviviridae) are transmitted from infected to susceptible humans primarily by Aedes mosquitoes. ${ }^{1}$ Following infection, an individual remains vulnerable to re-infection with a different serotype of the virus. Clinically-apparent disease usually occurs with the first or second infections, rarely with the third or fourth. ${ }^{2}$ There is a wide spectrum of clinical manifestations from asymptomatic to undifferentiated fever, dengue fever (DF), dengue haemorrhagic fever (DHF) and dengue shock syndrome (DSS). ${ }^{3}$

With an estimated 50 million infections annually, ${ }^{3}$ the burden of dengue is a heavy one indeed, ${ }^{4}$ especially to countries in the Asia Pacific region. ${ }^{5}$ Despite sustained vector control efforts, Singapore has also not been spared from the disease. Although the Aedes premises index (percentage of premises found breeding Aedes mosquitoes) has been consistently maintained at

a Hospital Services Division, Ministry of Health, Singapore

b Communicable Diseases Division, Ministry of Health, Singapore

c Environmental Health Institute, National Environment Agency, Singapore

dEnvironmental Health Department, National Environment Agency, Singapore

e Office of the Director of Medical Services, Ministry of Health, Singapore

Submission date: 29 November 2010; Publication date: 20 May 2011

doi: 10.5365/wpsar.2010.1.1.011 between $1 \%$ and $2 \%$ since the 1980 s, Singapore still experienced successive dengue outbreaks at intervals of five to seven years. ${ }^{6-10}$

In 2005, Singapore experienced its largest dengue outbreak on record. ${ }^{10}$ Only two years thereafter, another outbreak ensued. As this was not in keeping with the five-to-seven-year cycles previously experienced, we undertook epidemiological, entomological and virological studies to investigate the features of the 2007 dengue outbreak, and compared them with those of 2005 , to determine the factors that triggered the outbreak.

\section{METHODS}

\section{Dengue surveillance and control in Singapore}

In Singapore, the Ministry of Health is responsible for the epidemiological surveillance and clinical management of dengue. The National Environment Agency (NEA), 
Ministry of Environment and Water Resources takes charge of vector surveillance, control and research.

\section{Notification}

All medical practitioners are required by the Infectious Diseases Act to report all clinically-diagnosed and laboratory-confirmed dengue cases and deaths to the Ministry of Health by facsimile or by online electronic notification within 24 hours. They also are required to report cases that were initially diagnosed as DF but that later satisfied criteria for DHF. ${ }^{11}$ Directors of clinical laboratories also are mandated to notify the Ministry of Health whenever any blood sample provides evidence of recent dengue infection. Laboratory tests used in Singapore are RT-PCR, ${ }^{12}$ immunochromatographic assays, and commercial kits for the detection of NS1 or anti-DENV IgM. Epidemiological data routinely collected included name, identification number, age, ethnicity, gender, occupation, residential and workplace/school addresses and date of onset of illness.

\section{Vector control}

On receipt of notification, epidemiological data are immediately transmitted to the NEA, which undertakes field epidemiological investigations if necessary. All reported cases and breeding habitats are plotted, using a geographical information system, to determine clustering of cases and to identify high-risk areas for priority vector control. ${ }^{13}$

\section{Serotype surveillance}

The Environmental Health Institute (EHI) at the NEA, monitors the circulating DENV serotypes and genotypes using samples submitted by Tan Tock Seng Hospital and by a network of sentinel private medical practitioners. Detection of dengue virus RNA and serotyping was performed with an in-house real-time PCR. ${ }^{12}$ Phylogenetic analyses of the dengue virus envelope protein gene has been described elsewhere. ${ }^{14}$ Serotyping of dengue virus was also carried out at the Department of Pathology, Singapore General Hospital, National University Hospital and Kandang Kerbau Hospital.

\section{Data analysis}

Only laboratory-confirmed cases reported to the Ministry of Health were included in the analysis reported here; we further restricted these cases to those who were Singaporean citizens or permanent residents. All duplicate notifications were removed before analysis. Cases with a history of travel within seven days before the onset of illness to countries where dengue also occurred were classified as imported and were not included in the analysis. A cluster was defined as two or more cases within a 150 meter radius (based on residential or workplace/school addresses) and with the onset of illness within a 14-day period. Denominators for the calculation of incidence rates were based on the estimated mid-year population obtained from the Singapore Department of Statistics, Ministry of Trade and Industry. ${ }^{15}$

\section{Seroprevalence survey}

To determine the proportion of asymptomatic infections, voluntary blood sampling was carried out in 2007 in the three largest clusters of cases at Bukit Batok, Woodlands and Pasir Ris, where all household members residing in each site were invited to participate. A recruitment and sampling site was set up within each of the clusters to take blood samples $(3 \mathrm{ml})$ and to conduct face-toface interviews. Only residents who gave written consent were interviewed. Individuals whose serum samples contained anti-DENV IgM were scored as having a recent infection and those whose serum samples contained anti-DENV IgM but could not recall any recent symptoms of dengue infection were classified as asymptomatic. ${ }^{16}$

\section{Statistical analysis}

Microsoft Office Excel 2007 and SPSS 15.0 were used for statistical analyses. Differences between the agegender-standardized incidence rates were computed and tested for statistical significance using the Z-test. ${ }^{17}$ Statistical significance was taken at $P<0.05$ level.

\section{Ethics}

The study was conducted primarily as part of a national public health programme, with supplemental data obtained from a community seroprevalence study which had been approved by the National Environment Agency Bioethics Review Committee (IRB 005.2) in 2007.

\section{RESULTS}

\section{Epidemiological findings}

A total of 8826 laboratory-confirmed cases of dengue were reported in 2007, an incidence rate of 192.3 per 100000 population. Of these, 8637 (97.9\%) were DF and 189 (2.1\%) were DHF. Twenty-four fatalities were 
Figure 1. Monthly distribution of dengue cases in 2005 and 2007

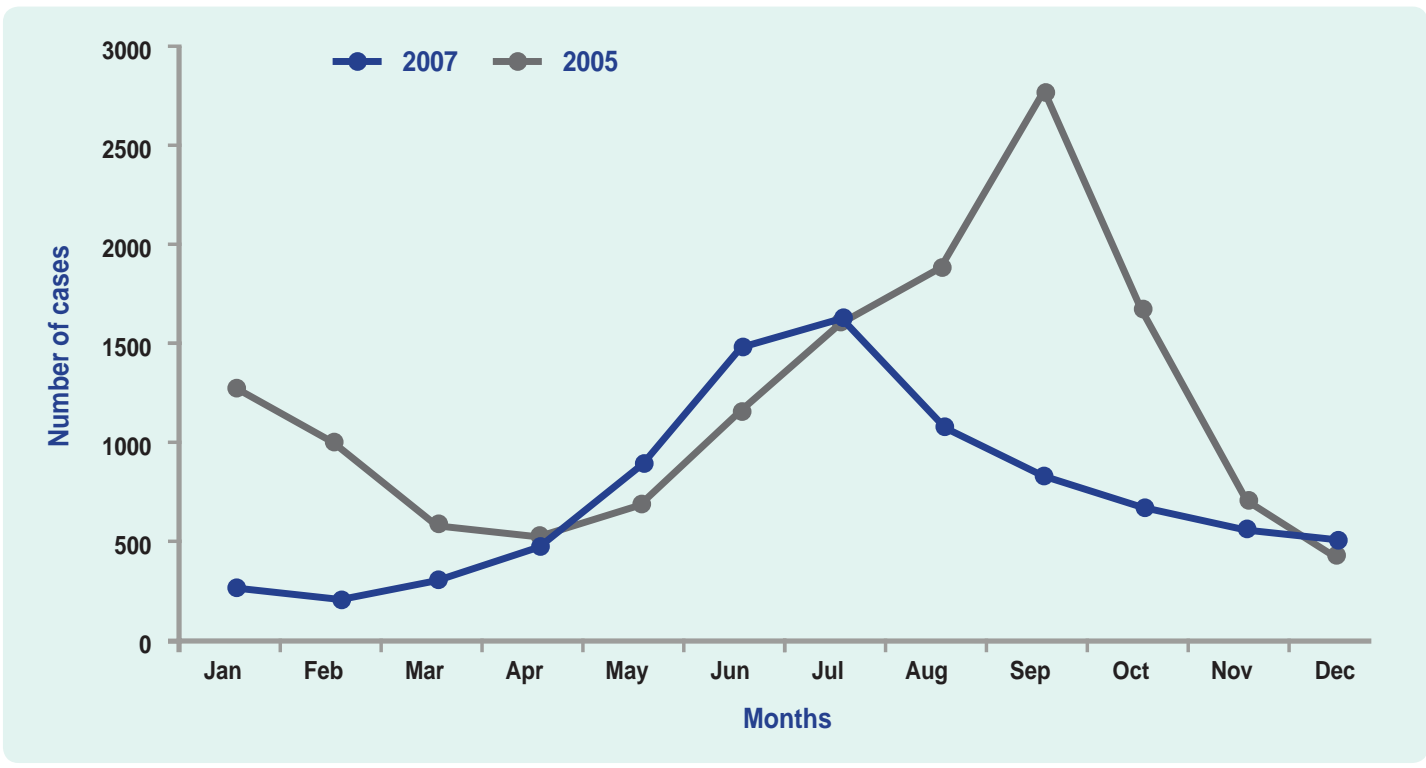

reported in 2007, comprised of eight DF and 16 DHF cases, a case-fatality rate (CFR) of $0.27 \%$ for all dengue cases and $8.5 \%$ for all DHF cases, comparable to the outbreak in 2005. In 2005, 27 fatalities were reported, comprising three DF and 24 DHF cases, a CFR of $0.19 \%$ for all dengue cases and $6.1 \%$ for all DHF cases. ${ }^{10}$ The outbreaks in 2005 and 2007 differed in that 257 cases of DF/DHF were reported in January 2007, far fewer than the 1262 cases reported in the same period of 2005. ${ }^{10}$ Most cases (1633) were reported in July 2007, two months earlier than the peak of 2770 cases that were reported in September $2005^{10}$ (Figure 1).

The median age of DF/DHF cases among Singapore residents in 2007 was 37 years (interquartile range 25 to 50), with a range from two days to 101 years, higher than the median age of 31 years (interquartile range 20 to 42), with a range from four days to 98 years, in 2005. ${ }^{10}$ The age-specific incidence rate for dengue was highest in the age group 55 years and above in 2007. In 2005 , it was highest in the 15 to 24 -year-old age group (Table 1). ${ }^{10}$

The incidence in males was significantly higher than in females (209.3 per 100000 and 150.3 per 100 000, respectively) and that was true for all ethnic groups $(P<0.01)$. Among Singapore residents in 2007 , the age-gender-adjusted incidence rate of DF/DHF was highest in the Chinese (172.3 per 100000$)(P<0.01)$, followed by the Malays (146.9 per 100000$)(P<0.05)$ and the Indians (120.9 per 100 000). Among Singapore residents in 2005, the age-gender-adjusted incidence rate of DF/DHF was also highest in the Chinese (312.8 per 100 000) followed by the Malays (288.4 per $100000)$ and the Indians (173.9 per 100000$).{ }^{10}$

The incidence rate in 2007 was highest among those residing in private condominiums (453.8 per

Table 1. Age-specific incidence rates of reported indigenous cases of dengue, Singapore, 2005 and 2007

\begin{tabular}{crrrrrr}
\hline \multirow{2}{*}{$\begin{array}{c}\text { Age group } \\
\text { (years) }\end{array}$} & Number & $\mathbf{( \% )}$ & $\begin{array}{r}\text { Incidence rate } \\
\text { (per 100 000) }\end{array}$ & Number & \begin{tabular}{r} 
(\%) \\
\cline { 2 - 7 }
\end{tabular} & $\begin{array}{r}\text { Incidence rate } \\
\text { (per 100 000) }\end{array}$ \\
\hline $0-4$ & 184 & $(1.3)$ & 86.9 & 100 & $(1.2)$ & 48.0 \\
$5-14$ & 1749 & $(12.5)$ & 334.3 & 530 & $(6.4)$ & 102.6 \\
$15-24$ & 3078 & $(21.9)$ & 474.3 & 1270 & $(15.3)$ & 176.7 \\
$25-34$ & 3253 & $(23.2)$ & 376.8 & 1802 & $(21.7)$ & 188.8 \\
$35-44$ & 2762 & $(19.7)$ & 362.8 & 1777 & $(21.4)$ & 219.7 \\
$45-54$ & 1587 & $(11.3)$ & 262.1 & 1134 & $(13.7)$ & 174.4 \\
$55+$ & 1419 & $(10.1)$ & 217.7 & 1674 & $(20.2)$ & 228.9 \\
Total & 14032 & $(100.0)$ & 328.9 & 8287 & $(100.0)$ & 180.6 \\
\hline
\end{tabular}




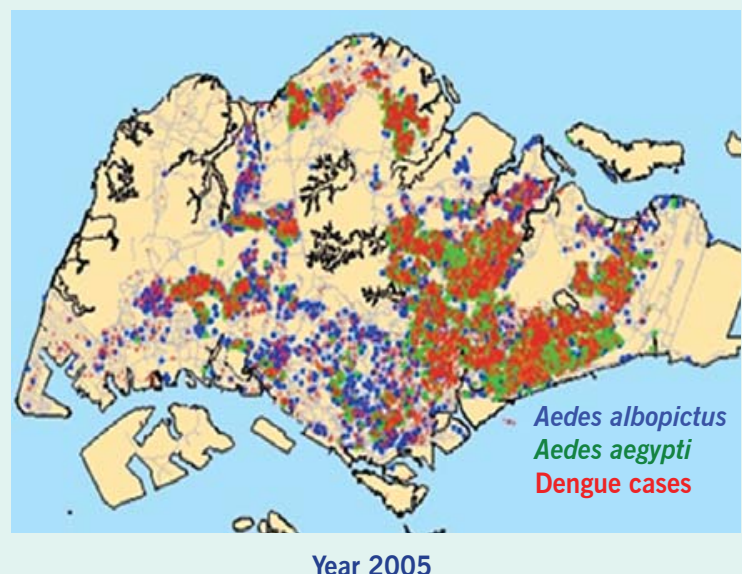

Year 2005

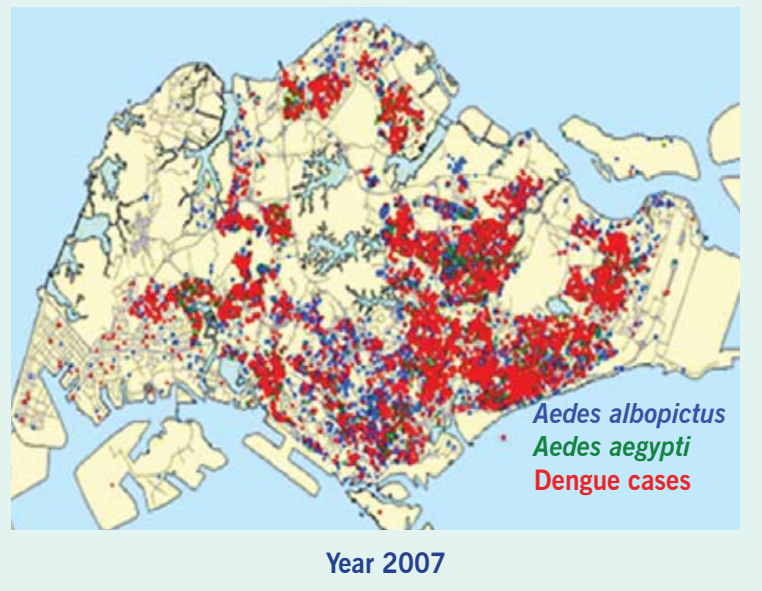

serotype circulating in 2005 was DENV-1, ${ }^{10}$ but in January 2007 it was overtaken by DENV-2, which re-emerged with a clade replacement, ${ }^{14}$ and has since remained the predominant serotype (Figure 3). In 2007, RNA from 1044 confirmed cases of acute dengue infection were serotyped at the EHI, NEA, Department of Pathology, Singapore General Hospital and the laboratories at Tan Tock Seng Hospital and National University Hospital, and comprised 88.3\% DENV-2, 6.4\% DENV-1, 4.6\% DENV-3 and 0.7\% DENV-4.

\section{Entomological findings}

As in 2005, the distribution of dengue cases in 2007 was more closely associated with Aedes aegypti than with Aedes albopictus breeding sites (Figure 2). ${ }^{10}$ The overall Aedes premises index was $0.68 \%$ in 2007 , lower than the $1.15 \%$ observed in $2005 .{ }^{10}$

The top three breeding habitats for Aedes aegypti in 2005 and 2007 were similar, with domestic containers (26\% in $2005,32 \%$ in 2007), ornamental containers (24\% in $2005,21 \%$ in 2007$)$ and flower pot plates (7\% in $2005,11 \%$ in 2007) constituting more than half of all breeding habitats. ${ }^{10}$ However, for Aedes albopictus, there was a decrease in the number of breeding sites found in discarded receptacles (from 21\% in 2005 to $4 \%$ in 2007). ${ }^{10}$

\section{DISCUSSION}

With the exception of median age of the cases, and the predominant dengue serotype, the epidemiological features of the 2007 epidemic were largely similar to those of 2005. The increase in median age and the 
Figure 3. Dengue virus serotypes identified at Environmental Health Institute, Department of Pathology, Singapore General Hospital and laboratories at Tan Tock Seng Hospital and National University Hospital, Singapore, 2005-2007

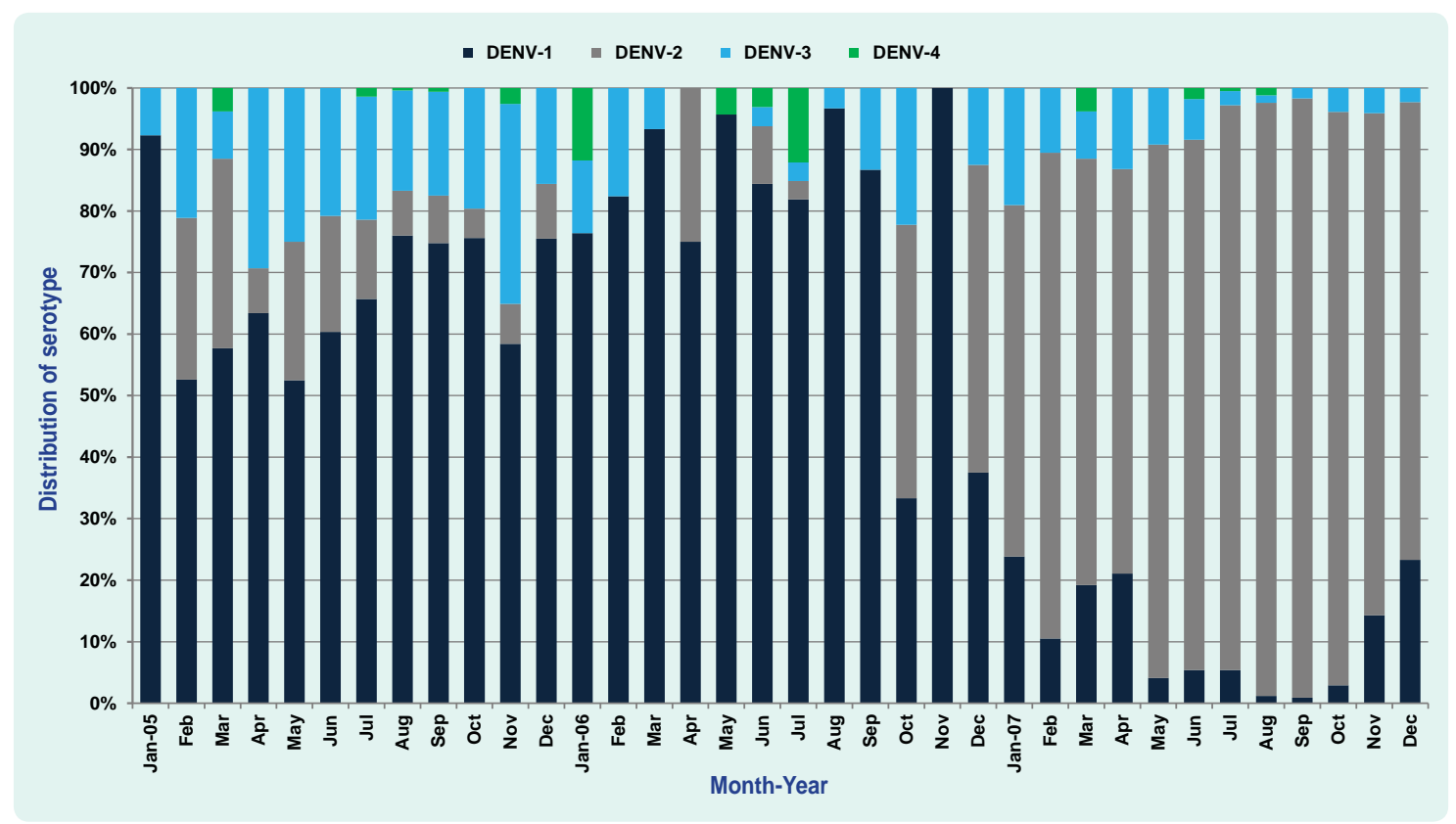

finding that those aged more than 55 years had the highest incidence are poorly understood. It is interesting to note that this phenomenon has accompanied the switch to DENV-2, and both the phenomenon and predominance of DENV-2 have persisted till 2010.

After the 2005 outbreak, the dengue control system was enhanced going into 2007 . First, manpower that routinely carried out Aedes surveillance and source reduction was increased from 250 in 2005 to about 500 in 2007. On average, 116764 residential premises and 3011 non-residential premises (e.g. construction sites, schools) were inspected every month in 2007, resulting in almost all premises being inspected within the year. ${ }^{13}$ Second, an intensive source reduction exercise was conducted by NEA officers two to three months before the expected dengue season, to remove breeding and potential breeding grounds. Third, early warnings systems were developed through virological surveillance and ambient temperature monitoring, providing temporal risk stratification. ${ }^{14}$ Lastly, qualitative spatial risk stratification was performed using case, virus and larva surveillance data, to guide the deployment of staff. Despite the enhanced vector control which led to a premises index of $0.68 \%$, which was significantly lower than the $2.24 \%, 1.7 \%$ and $2 \%$ found from 2002 to 2004 (when the same cosmopolitan genotype DENV was predominant), respectively, an outbreak still ensued.
Phylogenetic analyses of DENV-2 envelope gene sequences at $\mathrm{EHI}, \mathrm{NEA}$, revealed that the switch in predominant serotype in early 2007 coincided with a clade replacement within DENV-2. Within the Cosmopolitan genotype of DENV-2, there were two distinct subclades with strong temporal topology. The old clade was detected from 2000 to 2005 and the new clade from 2007 to $2008 .{ }^{14}$ The role of the small genetic changes between the two clades, in viral fitness, is being investigated. A similar observation was reported in Cuba, where the authors hypothesized that the 1997 severe outbreak in Cuba was a result of a small mutation that improved the fitness of DENV-2. ${ }^{18}$

The epidemiology of dengue in Singapore has evolved from a paediatric problem in the 1960s to an adult infection since the 1980s. The median age has shifted from 14 years in $1973,{ }^{19}$ to 37 years in 2007. This has been attributed to the lower transmission rate, which was demonstrated by a mathematical model that showed a declining trend in the force of infection (defined as per capita rate at which susceptible individuals in the community acquire infection) ${ }^{20}$ since the nationwide vector control programme was first implemented in the late 1960s.

Previous studies have demonstrated that more than $90 \%$ of dengue infections were asymptomatic. ${ }^{21}$ In a survey representative of the adult general population 
conducted in 2004, the prevalence of asymptomatic infections was found to be $95 \% .{ }^{22}$ The prevalence of asymptomatic dengue infections in surveys conducted during the 2007 outbreak in three public housing estates ranged from $57.1 \%$ to $81.8 \%$. The differences could be due to different methodologies.

A limitation on this study would be the reliance on notification data. The incidence rate is actually the case notification/detection rate. Although medical practitioners and directors of clinical laboratories were required to report all cases of DF/DHF to the Ministry of Health, mild cases with undifferentiated fevers may not seek medical care and so may not be reported. The actual incidence of dengue infection in 2007, therefore, could be higher than was reported. A limitation of the seroprevalance survey was that convenience sampling was done, and data collected may not be representative of the population. Nevertheless, it gives us an idea of the prevalence of asymptomatic infections in these outbreak areas.

The 2007 dengue epidemic in Singapore demonstrated the dynamic interactions of the virus, human host, vector and the environment, as evidenced by the resurgence caused by introduction of a new clade of DENV-2, despite sustained vector control efforts. Given the large estimated proportion of asymptomatic infections, the real extent of the problem could be much larger than what is currently known. Singapore remains vulnerable to major outbreaks of dengue through the constant introduction of viruses from travellers; and through local evolution of the virus.

\section{Conflicts of interest}

None declared.

\section{Funding}

None.

\section{References:}

1. Halstead SB. Pathogenesis of dengue: challenges to molecular biology. Science, 1988, 239:476-81. doi: 10.1126/ science.3277268 pmid:3277268

2. Sabin $A B$, Schlesinger RW. Production of immunity to dengue with virus modified by propagation in mice. Science, 1945, 101:640-642. doi:10.1126/science.101.2634.640 pmid: 17844088

3. Dengue: guidelines for diagnosis, treatment, prevention and control - New edition. Geneva, World Health Organization, 2009 (http://whqlibdoc.who.int/publications/2009/9789241547871 eng.pdf, accessed on 28 February 2011).
4. Suaya JA et al. Cost of dengue cases in eight countries in the Americas and Asia: a prospective study. The American Journal of Tropical Medicine and Hygiene, 2009, 80:846-855. pmid:19407136

5. The Dengue Strategic Plan for the Asia Pacific Region 20082015. New Delhi (SEARO) and Manila (WPRO), World Health Organization, 2008. (http://www.searo.who.int/LinkFiles/Dengue Dengue_Strategic_Plan_for_the_Asia-Pacific_Region_(20082015).pdf, accessed on 28 Fébruary 2011).

6. Goh KT et al. Epidemiological aspects of an outbreak of dengue fever/dengue haemorrhagic fever in Singapore. The Southeast Asian Journal of Tropical Medicine and Public Health, 1987, 18:295-302. pmid:3433161

7. Epidemiology of dengue fever/dengue haemorrhagic fever. World Health Organization, World Epidemiological Report, 1990 65:101-108. (http://whqlibdoc.who.int/wer/WHO_WER_1990/ WER1990 65 101-108\%20(N\%C2\%B014).pdf, accessed on 28 February $2 \overline{0} 11$ ).

8. Ministry of Health, Committee of Epidemic Diseases. Surveillance of dengue fever/dengue haemorrhagic fever, 1992. Epidemiological News Bulletin (Singapore), 1993, 19:13-17.

9. Ministry of Health, Committee of Epidemic Diseases. Dengue surveillance in Singapore, 1998. Epidemiological News Bulletin (Singapore), 1999, 25:1-3.

10. Koh BKetal. The 2005 dengue epidemic in Singapore: epidemiology, prevention and control. Annals of the Academy of Medicine, Singapore, 2008, 37:538-545. pmid:18695764

11. Goh KT, Ong A, Low J, editors. A guide on infectious diseases of public health importance in Singapore, 6th ed. Singapore, Ministry of Health and Tan Tock Seng Hospital, 2004.

12. Lai $Y L$ et al. Cost-effective real-time reverse transcriptase PCR (RT-PCR) to screen for dengue virus followed by rapid single-tube multiplex RT-PCR for serotyping of the virus. Journal of Clinical Microbiology, 2007, 45:935-941. doi:10.1128/JCM.0125806 pmid: 17215345

13. Tang CS et al. Surveillance and control of dengue vectors in Singapore. Epidemiological News Bulletin, 2006, 32: 1-9. (http://www.moh.gov.sg/dam/moh web/Statistics/ Epidemiological_News_Bulletin/2006/ENB01Q_06.pdf, accessed on 28 February 2011).

14. Lee KS et al. Dengue virus surveillance for early warning, Singapore. Emerging Infectious Diseases, 2010, 16:847-849. pmid:20409381

15. Singapore Resident Population 2003-2008. Singapore, Department of Statistics, 2009.

16. Yew YW et al. Seroepidemiology of dengue virus infection among adults in Singapore. Annals of the Academy of Medicine, Singapore, 2009, 38:667-675. pmid:19736569

17. Armitage P, Berry G. Statistical Methods in Medical Research, $2^{\text {nd }}$ ed. Oxford, Blackwell Scientific, 1987.

18. Rodriquez-Roche $\mathrm{R}$ et al. Virus role during intraepidemic increase in dengue disease severity. Vector-Borne and Zoonotic Diseases, 2011, 11:00.

19. Goh KT. Changing epidemiology of dengue in Singapore. Lancet, 1995, 346: 1098 . doi:10.1016/S0140-6736(95)91771-3 pmid:7564804

20. Egger JR et al. Reconstructing historical changes in the force of infection of dengue fever in Singapore: implications for surveillance and control. Bulletin of the World Health Organization, 2008, 86:187-196. doi:10.2471/BLT.07.040170 pmid: 18368205

21. Goh KT. Seroepidemiology of dengue virus infection in Singapore. In: Goh KT (ed). Singapore, Ministry of the Environment, 1998, 50-72.

22. Ye $T$ et al. Seroprevalence study on past and recent dengue virus infection in Singapore. Epidemiological News Bulletin (Singapore), 2007, 33:36-41 (http://www.moh.gov.sg/content/dam/moh web/ Statistics/Epidemiological_News_Bulletin/2007/ENB03Q_07.pdf, accessed on 28 February 2011 ). 